\title{
Métodos de diagnóstico não-invasivos para avaliação da insuficiência venosa dos membros inferiores
}

\author{
Noninvasive diagnostic methods to evaluate venous insufficiency \\ of the lower limbs
}

Orlando Adas Saliba Jr. ${ }^{1}$, Mariangela Giannini ${ }^{2}$, Hamilton Almeida Rollo ${ }^{3}$

\section{Resumo}

A avaliação clínica dos membros inferiores na insuficiência venosa por si só não identifica os sistemas envolvidos ou os níveis anatômicos, sendo necessários exames complementares. Esses exames podem ser invasivos ou não-invasivos. Os invasivos, como flebografia e pressão venosa ambulatória, apesar de terem boa acurácia, trazem desconforto e complicações. Dentre os não-invasivos, destacam-se: Doppler ultra-som de ondas contínuas, fotopletismografia, pletismografia a ar e mapeamento dúplex. O Doppler ultra-som avalia a velocidade do fluxo sangüíneo de maneira indireta. A fotopletismografia avalia o tempo de reenchimento venoso, fornecendo um parâmetro objetivo de quantificação do refluxo venoso. A pletismografia a ar permite quantificar a redução ou não da capacitância, o refluxo e o desempenho da bomba muscular da panturrilha. O dúplex é considerado padrão-ouro dentre os não-invasivos, porque permite uma avaliação quantitativa e qualitativa, fornecendo informações anatômicas e funcionais, dando avaliação mais completa e detalhada dos sistemas venosos profundo e superficial.

Palavras-chave: Diagnóstico, insuficiência venosa, ultra-som, pletismografia.

\section{Insuficiência venosa}

Insuficiência venosa crônica (IVC) pode ser definida como um conjunto de alterações que ocorrem na pele e no tecido subcutâneo, principalmente nos membros inferiores, decorrentes de uma hipertensão venosa de longa duração, causada pela insuficiência valvular e/ou obstrução venosa ${ }^{1}$.

Em 1994, no Fórum Venoso Americano realizado em Maui (Hawai, EUA), foi desenvolvida a classificação das

\begin{abstract}
Clinical evaluation of lower limbs of patients with venous insufficiency alone may not identify involved systems or anatomical sites, thus complementary tests are needed. These tests can be invasive or noninvasive. Invasive tests, such as phlebography and ambulatory venous pressure, despite being accurate, may produce discomfort and complications. Some of the most used noninvasive tests are continuous wave Doppler ultrasound, photoplethysmography, air plethysmography and duplex scanning. Doppler ultrasound assesses blood flow velocity indirectly. Photoplethysmography assesses venous refilling time, providing an objective parameter of venous reflux quantification. Air plethysmography allows quantification of reduction in venous capacitance, reflux and performance of the calf muscle pump. Duplex is considered a gold standard among noninvasive methods, because it allows quantitative and qualitative evaluation, supplying anatomical and functional information, thus providing a more complete and detailed evaluation of both deep and superficial venous system.
\end{abstract}

Keywords: Diagnosis, venous insufficiency, ultrasonics, plethysmography.

doenças venosas baseada em sinais clínicos (C), etiologia (E), distribuição anatômica (A) e disfunção fisiopatológia (P), denominada classificação $\mathrm{CEAP}^{2}$. Considerada classificação padrão, possibilita uniformidade no relato e avaliação de diferentes modalidades de diagnóstico e tratamento. Em 2004, foi proposta uma modificação nessa classificação, com vistas a seu aperfeiçoamento ${ }^{3}$. As alterações que compõem o que se denomina IVC são as incluídas nas classes clínicas de 2 a 6 da classificação CEAP $^{2}$.

\footnotetext{
1. Mestre, Faculdade de Medicina de Botucatu (FMB), Universidade Estadual Paulista (UNESP), Botucatu, SP.

2. Doutora. Professora assistente, Disciplina de Cirurgia Vascular, Departamento de Cirurgia e Ortopedia, FMB, UNESP, Botucatu, SP.

3. Doutor. Professor assistente, Disciplina de Cirurgia Vascular, Departamento de Cirurgia e Ortopedia, FMB, UNESP, Botucatu, SP.
}

Artigo submetido em 09.11.06, aceito em 14.06.07. 
A IVC é uma afeccção muito comum, embora de mortalidade praticamente nula, apresenta morbidade importante, leva à piora da qualidade de vida dos doentes e tem impacto socioeconômico muito grande, inclusive em nosso país ${ }^{1}$.

Em estudo epidemiológico realizado no Brasil por Maffei et al. ${ }^{4}$, incluindo pacientes que buscaram atendimento em um Centro de Saúde Escola de Botucatu (SP) para exames de rotina, foi observada prevalência de varizes de 47,6\%. Com a correção estatística, a prevalência estimada para a população de mesmo nível socioeconômico do município foi de $35,5 \%$, excluindo os casos de queixas relativas aos membros inferiores.

González-Fajardo et al. ${ }^{5}$ citam um estudo patrocinado pela Organização Mundial da Saúde (OMS) na Espanha, no qual foi observada prevalência de 10,5\% de doenças venosas de membros inferiores em uma amostra de 4.800 pessoas de 30 a 65 anos de idade.

Barros Jr. ${ }^{6}$ realizou um estudo com pacientes gestantes do serviço pré-natal do Hospital Amparo Maternal, com o objetivo de analisar a prevalência da doença varicosa, fatores de risco e sintomatologia durante a gestação. O estudo mostrou alta prevalência da doença varicosa de $72,7 \%$, sendo que os fatores de risco mais associados foram a idade e antecedente familiar positivo para varizes.

Scuderi et al. ${ }^{7}$ avaliaram os achados clínicos das doenças venosas em 2.104 indivíduos, de acordo com o código $\mathrm{C}$ da classificação CEAP. Os indivíduos foram categorizados segundo sexo e idade, e as mulheres segundo o número de gestações. Na faixa etária de 14 a 22 anos, 46,42\% não tinham sintomas ou veias aparentes, ao passo que, na faixa etária acima de 48 anos, apenas $4,67 \%$ apresentaram essa condição. A maioria das mulheres $(62,79 \%)$ tinha sintomas e veias aparentes, ao passo que, nos homens, a maior parte $(65,54 \%)$ não apresentava sintomas ou veias aparentes. Os autores concluíram que a prevalência é muito maior no sexo feminino que no masculino e que a idade e o número de gestações foram fatores importantes no desenvolvimento da doença.

As doenças venosas apresentam características peculiares, pois algumas delas, com alterações superficiais, podem ser percebidas pelos próprios pacientes; entretanto, outras alterações envolvendo sistema venoso profundo (SVP) em estágios iniciais freqüentemente não provocam sintomas ou sinais que revelem sua existência ${ }^{8}$.

A avaliação clínica dos indivíduos com insuficiência venosa dos membros inferiores, apesar de ser importante, por si só não identifica os sistemas envolvidos ou os níveis anatômicos ${ }^{9,10}$. As úlceras provocadas pela IVC podem resultar de obstrução ou refluxo no SVP, de refluxo nas veias do sistema superficial e nas veias perfurantes ou da combinação desses.

Para que a avaliação seja mais detalhada e precisa, além do exame clínico, devem ser empregados exames complementarese, que podem utilizar métodos ou testes invasivos e não-invasivos.

Os testes invasivos que têm sido utilizados são a flebografia e a medida direta da pressão venosa ambulatória (PVA), por punção de uma veia no dorso do pé. A flebogafia, que tem sido considerada o método padrãoouro, permite a visualização do sistema venoso e identifica alterações morfológicas e funcionais ${ }^{9,11}$. Com isso, proporciona informações anatômicas e fisiopatológicas importantes não só para o diagnóstico, mas também para escolha de técnicas cirúrgicas ou endovasculares para correção da IVC. A PVA faz uma avaliação global da IVC, mas não identifica se as alterações são por obstrução ou refluxo no SVP ${ }^{11}$. A flebografia e a PVA, por serem invasivas, podem trazer desconforto e complicações para os pacientes. Assim, esses testes têm baixa aceitação e fica difícil sua repeticão para o seguimento de pacientes ou avaliação de terapêuticas para a IVC.

Com o intuito de fazer o diagnóstico de modo nãoinvasivo e confiável, ao longo das últimas décadas do século passado, alguns métodos não-invasivos foram desenvolvidos para avaliação da função venosa em pacientes com insuficiência venosa, como Doppler ultrasom de ondas contínuas (DOC), fotopletismografia (FPG), pletismografia a ar (PGA) e mapeamento dúplex (MD), entre outros $2,8,11-14$.

Os testes não-invasivos são mais econômicos, rápidos e não causam tanto desconforto aos pacientes quando comparado com os invasivos ${ }^{15}$. 
Serão descritos, a seguir, os principais métodos nãoinvasivos e suas capacidades de diagnóstico da IVC.

\section{Doppler ultra-som de ondas contínuas}

O DOC foi desenvolvido por Satomura \& Kaneko, em $1960^{16}$, e avalia a velocidade do fluxo sangüíneo arterial ou venoso por meio da detecção da mudança ou variação na freqüência do feixe de ultra-som refletido, a partir das células vermelhas em movimento.O instrumento produz um sinal sonoro audível ou uma forma de onda que pode ser registrável. O padrão de normalidade do fluxo venoso é de som espontâneo e fásico com a respiração. É audível com a sonda Doppler em todas as localizações, exceto nas veias superficiais e de menor calibre $^{17}$.

As principais vantagens do DOC são seu baixo custo, facilidade de transporte e possibilidade de repetição do exame quantas vezes for necessário. Como desvantagens, pode-se citar a subjetividade da técnica, exigindo muito tempo de treinamento para o examinador conseguir realizar o exame com facilidade e exatidão ${ }^{17,18}$. Por se tratar de um método qualitativo e não quantitativo, quando o acompanhamento não é realizado pelo mesmo profissional, a avaliação fica ainda mais difícil ${ }^{19-22}$.

Lucas et al. ${ }^{23}$ avaliaram 210 membros inferiores por meio da ausculta no trajeto da veia safena interna com o Doppler, durante manobra de Trendelemburg. Em 10 membros com varizes primárias, foram feitos também exames de flebografia, com o objetivo de confrontar os resultados, e a correlação foi observada em todos os casos. Cem membros inferiores que apresentaram varizes primárias foram classificados segundo a extensão e o calibre das varizes; os outros 100 membros inferiores não apresentaram varizes clinicamente detectáveis. Os autores observaram insuficiência da veia safena interna em 57,3\% das 89 extremidades com varizes primárias bilateral e 18,18\% nas 11 extremidades normais dos pacientes com varizes unilateral. Nos 100 membros não portadores de varizes, $2 \%$ apresentaram, ao exame, insuficiência da veia safena.

Bartolo et al. ${ }^{24}$ estudaram 9.178 membros inferiores e avaliaram a pressão venosa com o DOC. Verificaram que os valores da pressão venosa em repouso aumentaram gradativamente dos membros normais para os varicosos, atingindo níveis mais elevados nos portadores de síndrome pós-flebítica.

Menezes \& Sales ${ }^{19}$ avaliaram 96 membros inferiores de 50 pacientes com insuficiência venosa utilizando DOC. Verificaram a presença de alterações no SVP em 14 pacientes $(28 \%)$, três deles com lesões bilaterias, totalizando 17 membros com alterações (17,7\% dos 96 membros). O refluxo foi observado em $9,37 \%$ dos membros, mostrando-se associado à presença de complicações clínicas das varizes, e a obstrução em 8,33\% não esteve associada a um quadro clínico mais grave.

Cheng et al. ${ }^{25}$ utilizaram o DOC e a FPG na avaliação de 1.583 membros de 878 pacientes que apresentaram sintomas de IVC. A combinação de insuficiência das valvas dos sistemas superficial e perfurantes foi encontrada na maioria dos pacientes. Os autores consideraram que os resultados dos exames empregados foram úteis na avaliação dos pacientes.

Um estudo realizado no laboratório do Hospital das Clínicas da Faculdade de Medicina de Botucatu (FMB) avaliou 71 membros inferiores de 50 pacientes, portadores de varizes e/ou IVC, utilizando o DOC em comparação com MD na avaliação do refluxo venoso ou incompetência valvar ${ }^{26}$. Os resultados encontrados foram: sensibilidade de 71 e $78 \%$, especificidade de 87 e $81 \%$ e acurácia de 84 e $79 \%$, respectivamente, para as veias poplítea e safena magna.

Pelo descrito acima, o DOC pode ser utilizado na prática diária do angiologista e cirurgião vascular como um instrumento auxiliar ao exame clínico dos pacientes com insuficiência venosa, permitindo selecionar aqueles que deverão ser encaminhados para testes diagnósticos mais acurados, como o MD. Assim, o DOC, em mãos de examinadores experimentados, pode dar informações importantes sobre a presença ou não do refluxo venoso.

\section{Fotopletismografia}

A FPG, introduzida em estudos hemodinâmicos venosos por Barnes et al. ${ }^{27}$, é um método de avaliação de função venosa de fácil execução, que não requer treinamento demorado do operador e que avalia o tempo 
de reenchimento venoso (TRV), fornecendo um parâmetro objetivo de quantificação do refluxo venoso ${ }^{28}$. É usada em alguns serviços para diagnóstico de doenças venosas dos membros inferiores e acompanhamento de pacientes candidatos a cirurgia de varizes no pré e pós-operatório ${ }^{6,28-34}$.

Os valores absolutos encontrados nos exames de FPG no pré-operatório e no pós-operatório são medidas quantitativas objetivas úteis para verificação da mudança hemodinâmica venosa resultante da cirurgia de varizes. Possibilitam verificar se a cirurgia de varizes melhorou ou não a hemodinâmica do membro do paciente ${ }^{28}$.

O TRV é medido e registrado em uma curva a partir de uma linha de base. O valor do TRV é uma medida indireta do retorno venoso e da incompetência das val$\operatorname{vas}^{35}$. Muitos pesquisadores utilizaram a FPG para avaliação da doença venosa dos membros inferiores ${ }^{31,34,36-39}$ e seguimento pós-operatório, com a finalidade de verificar a eficácia cirúrgica ${ }^{25,40-44}$. A maioria dos autores considera como TRV normais valores acima de $20 \mathrm{~s}$, como Gaitini et al. ${ }^{45}$, Evangelista \& Fonseca ${ }^{28}$, Sakurai et al. ${ }^{33} \mathrm{e}$ Welch et al. ${ }^{11}$. Outros pesquisadores, como Sanches \& Gomes $^{32}$, Gonzáles-Fajardo ${ }^{6}$ e Lane et al. $^{44}$, consideram valores normais aqueles acima de $23 \mathrm{~s}$.

Em casos de reenchimento do sistema venoso através de valvas incompetentes (refluxo), há um retorno anormalmente rápido da curva do exame à linha de base inicial ${ }^{35}$. A FPG é útil para avaliar o refluxo do sistema venoso superficial nos pacientes com SVP normal, pois não avalia bem o grau de refluxo no profundo ${ }^{46}$.

Também pode-se realizar o exame com colocação de garrotes ou manguitos de pressão de insuflação automática acoplados ao aparelho, em posição que impedem o refluxo do sistema venoso superficial (safenas magna e parva), obtendo-se valores preditivos para a diminuição do refluxo com a cirurgia venosa no sistema superficial $^{46,47}$. A medida do refluxo baseia-se no esvaziamento venoso provocado por contrações na panturrilha em virtude da realização de exercícios, podendo ser afetada pelos seguintes fatores:
- Obstruções em veias profundas podem impedir o esvaziamento adequado da panturrilha, o que pode provocar um tempo de reenchimento reduzido e que será interpretado como refluxo.

- Incapacidade do paciente em realizar os movimentos com os pés de forma adequada (problemas neurológicos, artrites, anquilose, etc.) impede o esvaziamento adequado da panturrilha, o que provocará um tempo de reenchimento reduzido, podendo ser interpretado como refluxo.

- Pacientes com grandes níveis de hiperemia provocada por exercício apresentarão um TRV mais rápido, que poderá parecer refluxo ${ }^{47}$.

Sarin et al. ${ }^{30}$ compararam curvas do exame de FPG com a avaliação clínica e com o exame de MD em pacientes com insuficiência venosa e constataram uma boa correlação nos resultados observados. Os pacientes com insuficiência venosa grave apresentaram invariavelmente dados bastante alterados de TRV, sendo esse parâmetro de avaliação considerado confiável.

Sanches \& Gomes ${ }^{32}$, em 1991, estudaram 35 membros inferiores em 18 indivíduos sem antecedentes de enfermidade venosa, com o objetivo de estabelecer uma rotina clínica para o exame de FPG. Foram estabelecidas etapas de acordo com o valor do TRV, permitindo, assim, a estimativa dos setores venosos. Foram considerados resultados normais aqueles cujos valores eram $\geq \mathrm{a}$ $23 \mathrm{~s}$. Os autores concluíram que a rotina clínica elaborada permite o diagnóstico da insuficiência valvar quando um ou vários segmentos estão afetados.

Tucker et al. ${ }^{38}$ realizaram estudo com a FPG para avaliação de pacientes normais, pacientes que apresentavam dor na perna, com ou sem IVC. O método apresentou-se bastante reprodutível.

Dunn et al. ${ }^{41}$ empregaram a FPG para avaliação pré e pós-operatória de seis pacientes submetidos a transplante valvar de membros inferiores. Esses pacientes apresentavam TRV alterado $(<20 \mathrm{~s})$ e, após a cirurgia, houve uma melhora no TRV de $100 \%$ ou mais.

Rodriguez et al. ${ }^{43}$ utilizaram a FPG em pacientes com úlceras venosas de membros inferiores no pré e pósoperatório de valvuloplastia externa. Foi observado aumento significativo do TRV no pós-operatório, 
quando da cicatrização da úlcera, ocorrida entre 18 e 35 dias.

Froneck et al. ${ }^{39}$, em 2000, propuseram uma modificação na técnica convencional da FPG, permitindo quantificar o volume sangüíneo drenado induzido pela elevação do membro inferior acima do nível do coração, afetando a pressão hidrostática da perna. Atingido um patamar no registro gráfico, o membro é rapidamente abaixado e deixado em posição de relaxamento, causando, assim, o reenchimento venoso devido ao aumento da pressão hidrostática. Os autores concluíram que essa técnica permitiu avaliar a presença ou ausência de trombose venosa profunda e insuficiência das valvas do SVP.

A FPG tem sido usada como um método de acompanhamento em muitos laboratórios vasculares, pelo fato de o TRV ser um sensível indicador de refluxo, porém ela é incapaz de diferenciar graus de gravidade clínica da doença ${ }^{48}$.

Scriven et al. ${ }^{31}$, em estudo longitudinal prospectivo, realizaram exames fotopletismográficos no pré e pósoperatório de 30 dias para avaliar a eficácia da cirurgia de veias perfurantes. Foram submetidos a cirurgia sete membros ulcerados, que apresentavam insuficiência das veias perfurantes e das veias profundas. Os autores notaram que a FPG demonstrou anormalidades do refluxo venoso nos membros inferiores no pré-operatório, que persistiram após as cirurgias, sugerindo que a cirurgia de veias perfurantes isoladamente não trata adequadamente os pacientes com úlceras em membros inferiores e que apresentam insuficiência de veias profundas simultaneamente.

Desse modo, pelo que foi apresentado acima, a FPG ainda tem sido usada e tem-se mostrado um método útil para avaliação da IVC, bem como para avaliação de técnicas cirúrgicas ou outros procedimentos para tratar a insuficiêcia venosa.

\section{Pletismografia a ar}

A PGA é um método não-invasivo relativamente novo, utilizado para avaliação quantitativa do sistema venoso de membros inferiores e perfusão arterial $^{49}$ e que permite quantificar a redução ou não da capacitância venosa, o refluxo e o desempenho da bomba muscular da panturrilha ${ }^{50}$. Os equipamentos de PGA fazem esta quantificação pela determinação dos seguintes parâmetros: volume venoso (VV), volume ejetado por um tiptoe (VE), volume residual médio (VR) e tempo de enchimento até atingir 90\% de VV (TEV90). A partir desses parâmetros, são calculados os seguintes índices: índice de enchimento venoso (IEV), fração de ejeção (FE) e fração de volume residual (FVR).

Como vantagens, tem-se um exame cujos equipamentos e manutenção não são muitos dispendiosos, é menos examinador-dependente, de realização mais simplificada e, mesmo não fornecendo as informações anatômicas de maneira tão precisa quanto o MD, permite o seguimento dos pacientes para avaliação do tratamento realizado ${ }^{51,52}$.

Christopoulos et al. ${ }^{53}$ compararam PGA com a medida da pressão venosa ambulatorial em uma pesquisa para avaliar o efeito do uso de meias elásticas de média e alta compressão, respectivamente, para pacientes com insuficiência venosa superficial e profunda. Os autores verificaram a reprodutibilidade da PGA e concluíram que ela não é somente um método de valor diagnóstico, mas também possibilitou avaliar os efeitos hemodinâmicos de diferentes formas de compressão elástica.

Em outro estudo, Christopoulos et al. ${ }^{54}$ observaram uma boa correlação $(r=0,81)$ dos parâmetros da PGA com a medida da pressão venosa ambulatorial, analisando 30 membros normais, 110 com varizes primárias e 65 com oclusão ou insuficiência do SVP. Concluíram que a PGA é um método de boa acurácia para identificação do fator hemodinâmico predominante (alteração da bomba muscular da panturrilha e refluxo ou ambos), responsável pelo quadro clínico do paciente.

A PGA também é utilizada para monitoramento de resultados de cirurgias venosas. Christopoulos et al. ${ }^{55}$ avaliaram os resultados das cirurgias de 42 membros de 33 pacientes com varizes primárias no pré e pósoperatório de 8 a 10 dias por meio da PGA. O controle do refluxo venoso foi observado em todos os pacientes, e os autores concluíram que o método era objetivo e de grande valor diagnóstico. 
Gillespie et al. ${ }^{56}$ também utilizaram a PGA na avaliação de 25 membros inferiores antes e após a cirurgia de varizes, definidas de acordo com os exames clínicos e o MD. O método mostrou-se eficaz na avaliação de mudanças hemodinâmicas, especificamente quanto ao IEV e VV.

Estudos foram realizados com o intuito de verificar a correlação da PGA com o MD e com a flebografia ${ }^{11,13,14,57}$. Van Bemmelen et al. ${ }^{13}$ avaliaram 32 membros inferiores de 28 pacientes e obtiveram correlação pobre entre os achados do IEV da PGA e a presença de veias insuficientes e seus diâmetros verificados pelo MD. Porém, Weingarten et al. ${ }^{14}$ obtiveram bons resultados com a PGA na avaliação de 122 membros de 61 pacientes com diferentes graus de insuficiência venosa, com correlação estatisticamente significante entre o IEV da PGA e o tempo de refluxo determinado pelo MD.

Welch et al. ${ }^{11}$ concluíram, em estudo com 28 membros inferiores, que a PGA, quando comparada com a flebografia, foi capaz de detectar a presença de insuficiência venosa, porém não foi capaz de diferenciar os graus de gravidade da doença. Kalodiki et al. ${ }^{58}$, em estudo retrospectivo com 224 membros que tiveram sinais sugestivos de trombose venosa profunda e 41 membros sem veias varicosas e sem evidência de TVP na venografia, compararam a PGA com o método padrão-ouro (flebografia) e observaram que não houve diferença entre os dois métodos.

Yang et al. ${ }^{59}$ testaram a variabilidade dos parâmetros da PGA em pacientes com IVC repetindo os exames nos pacientes em diferentes ocasiões e notaram que havia variações nas determinações que foram repetidas em um mesmo paciente. Segundo esses autores, este resultado sugere ser pouco provável que a PGA seja capaz de detectar pequenas variações nos parâmetros estudados.

Entre nós, Engelhorn et al. ${ }^{60}$ objetivaram, em estudo com 88 membros de 48 pacientes, determinar quais os parâmetros da PGA que permitiriam diferenciar as classes leve, moderada e grave da IVC, de acordo com a classificação clínica CEAP. Os pacientes foram divididos em três grupos: grupo A - insuficiência venosa leve (classes 1 e 2); grupo B - insuficiência venosa moderada (classe 3); e grupo $\mathrm{C}$ - insuficiência venosa grave (classes $4,5 \mathrm{e}$
6). Não observaram diferença estatisticamente significante entre os graus leve e moderado de IVC, porém houve diferença entre o grupo $\mathrm{C}$, quando comparado com os grupos A e B.

Já Seidel ${ }^{61}$ estudou 100 membros de 81 pacientes, procurando correlacionar os exames de PGA com achados clínicos e com MD codificado em cores no diagnóstico da insuficiência da veia safena magna. Não foi possível avaliar a correlação da PGA com quadro clínico, pois a maioria $(61 \%)$ dos pacientes pertenciam à classe 2 da CEAP. A correlação dos resultados da PGA com os do MD foi muito fraca.

Assim, pode-se verificar que a maioria dos estudos com a PGA tem mostrado que ela é útil na avaliação da IVC, apesar de alguns autores ${ }^{13,59}$ ainda questionarem sua validade, pois a PGA não tem se mostrado um método capaz de identificar pequenas diferenças.

\section{Mapeamento dúplex}

O MD é uma combinação da ultrassonografia (US) modo B com a técnica do Doppler pulsado, permitindo, assim, uma avaliação simultânea da imagem bidimensional dos vasos e tecidos adjacentes e das características do fluxo através da análise da curva espectral Doppler ${ }^{62}$.

A imagem modo B é usada para avaliação anatômica da presença de trombo intraluminal ou alterações da parede e valvas venosas, e o Doppler pulsado permite detectar a presença do refluxo devido à incompetência valvular ${ }^{26}$. Em varizes dos membros inferiores, o exame de MD permite avaliar alterações no SVP, no superficial e nas veias perfurantes. Assim, possibilita diferenciar os pacientes com varizes primárias ou essenciais, cujas alterações ocorrem predominantemente no sistema venoso superficial, daqueles pacientes com varizes secundárias, em que o SVP é a sede das alterações patológicas. Deste modo, esse teste diagnóstico tem potencial que possibilita a avaliação tanto da anatomia quanto da fisiologia venosa ${ }^{62}$.

No MD em cores, os tecidos estáticos são mostrados em escala cinza, ao passo que o efeito Doppler é codificado em cores. A codificação em cores do fluxo, vermelho ou azul, depende da direção do fluxo em relação à orientação do feixe sonoro ${ }^{17}$. Este recurso facilita o 
exame de US, pois permite uma melhor visualização dos vasos, principalmente os de menor diâmetro, tornando o exame mais rápido.

O MD tem sido considerado um exame de acurácia semelhante à flebografia na identificação do refluxo venoso produzido por insuficiência valvular do sistema profundo, superficial e veias perfurantes ou combinação deles ${ }^{57}$. Pode também detectar pequenos refluxos em segmentos isolados em pacientes assintomáticos, e também se o refluxo está localizado em veias acima ou abaixo do joelho ou se extende por todo o membro. $\mathrm{Na}$ ausência de obstrução venosa profunda, membros com refluxo confinado nas veias acima do joelho raramente desenvolvem alterações de pele ou ulceração. O padrão de refluxo envolvendo no mínimo dois sistemas venosos (superficial e profundo; superficial e perfurantes; superficial, perfurante e profundo) é encontrado em cerca de $2 / 3$ de pacientes com alterações de pele ou ulceração ${ }^{63}$. O MD tem sido considerado não somente o mais específico exame não-invasivo, mas o mais facilmente aceito pelo paciente.

Neglen \& Raju ${ }^{64}$ avaliaram o refluxo em 56 membros de pacientes com insuficiência venosa por meio do $\mathrm{MD}$ comparado à flebografia. $\mathrm{O}$ valor preditivo positivo do dúplex foi de $77 \%$, comparado com 35 a $44 \%$ da flebografia descendente, comprovando a melhor acurácia do primeiro. Já Baker et al. ${ }^{65}$ compararam os achados do refluxo obtidos com o MD e a flebografia ascendente, concluindo que o MD foi mais sensível na detecção do refluxo venoso superficial e profundo e, em nível de coxa, permitiu também avaliar segmentos isolados com refluxo e também refluxo na safena parva.

Delis et al. ${ }^{66}$ utilizaram o MD para avaliação da hemodinâmica de veias perfurantes na insuficiência venosa de membros inferiores. Os resultados dos exames foram relacionados aos graus da classificação CEAP. Os autores afirmaram que nem o diâmetro das perfurantes, nem o tempo de refluxo e outros parâmetros hemodinâmicos investigados tiveram correlação com as classes CEAP, indicando que a gravidade da insuficiência venosa não depende primariamente da função isolada da perfurante, mas de múltiplos fatores, ocorrendo simultaneamente no sistema venoso.
Engelhorn et al. ${ }^{60}$, em estudo retrospectivo com 500 membros de 301 pacientes portadores de varizes primárias, realizaram exames de MD no pré-operatório, com o propósito de analisar uma nova classificação da insuficiência venosa superficial. Os autores ressaltam a importância da padronização da classificação da insuficiência venosa superficial para o planejamento cirúrgico, evitando possíveis recidivas ou a retirada desnecessária das veias safenas.

O MD é considerado o exame padrão-ouro dentre os não-invasivos, porque permite identificar e localizar as alterações fisiopatológicas, obstruções ou refluxos, tanto no SVP como no superficial, tendo nos últimos anos substituído a flebografia em muitas das suas indicações ${ }^{62}$. No entanto, é um exame demorado, examinador-dependente, que requer um tempo maior de treinamento e com custo mais elevado, pois os equipamentos são mais sofisticados e de manutenção mais cara. Por isso, sua utilzação para seguimento a longo prazo de pacientes com IVC fica dificultada, especialmente se os exames de seguimento devem ser feitos com freqüência.

\section{Considerações finais}

Nenhum teste diagnóstico não-invasivo feito isoladamente permite uma avaliação completa de todos os parâmetros anatômicos e funcionais que devem ser considerados na insuficiência venosa de membros inferiores.

O MD, considerado padrão-ouro dentre os métodos de diagnóstico não-invasivos para doenças venosas de membros inferiores, é atualmente o mais indicado por permitir avalição quantitativa e qualitativa. Fornece informações tanto anatômicas quanto funcionais, permitindo, assim, uma avaliação mais completa e detalhada do sistema venoso.

Nos casos em que não é possível a realização do MD, por inexistência de aparelho ou restrições em função de custo do exame, o profissional deve lançar mão da FPG ou da PGA para auxiliar no diagnóstico e acompanhamento do paciente com doença venosa. Essas pletismografias também são um método bastante útil na avaliação da doença venosa, bem como no acompanhamento de cirurgias de membros inferiores. 


\section{Referências}

1. Maffei FHA. Insuficiência venosa crônica: conceito, prevalência, etiopatogenia e fisiopatologia. In: Maffei FHA, Lastória S, Yoshida WB, Rollo HA, organizadores. Doenças vasculares periféricas. $3^{\mathrm{a}}$ ed. Rio de Janeiro: Medsi; 2002. p.1581-90.

2. Nicolaides NA. Consenso de classification de las enfermidades venosas crônicas. Patol Nasc. 1994;1:75-85.

3. Eklöf B, Rutherford RB, Bergan JJ et al. Revision of the CEAP classification for chronic venous disorders: Consensus statement. J Vasc Surg. 2004;40(6):1248-52.

4. Maffei FH, Magaldi C, Pinho SZ et al. Varicose veins and chronic venous insufficiency in Brazil: prevalence among 1755 inhabitants of a country. Int J Epidemiol. 1986; 15(2):210-7.

5. Gonzales-Fajardo JA, Rodriguez-Camarero SJ, Mareno P, et al. Valoración mediante fotopletismografía del efecto de una medicación venotônica. Angiologia. 1990;42(5):167-71.

6. Barros Jr N. Doença varicosa dos membros inferiores na gestação. Prevalencia, fatores de risco e sintomatologia [tese]. São Paulo: Universidade Federal de São Paulo, Escola Paulista de Medicina; 1995.

7. Scuderi A, Raskin B, Al Assal F et al. The incidence of venous disease in Braszil based on the CEAP classification. Int Angiol. 2002;21(4):316-21.

8. Maffei FHA. Diagnóstico clínico das doenças venosas periférica. In: Maffei FHA, Lastória S, Yoshida WB, Rollo HA, organizadores. Doenças vasculares periféricas. $3^{\mathrm{a}}$ ed. Rio de Janeiro: Medsi; 2002. v. 1, p.457-69.

9. Darke SG, Andress MR. The value of venography in the management of chronic venous disorders of the lower limb. In: Greenhalgh RM, editor. Diagnostic techniques and assessment procedures in vascular surgery. London: Grune \& Stratton; 1985. p.421-46.

10. McEnroe CS, O'Donnell TF, Mackey WC. Correlation of clinical finding with venous hemodynamics in 386 patients with chronic venous insufficiency. Am J Surg. 1998,156(2):148-52.

11. Welch HJ, Faliakou EC, McLaughlin RL, Umphrey SE, Belkin M, O'Donnell TF Jr. Comparison of descending phlebography with quantitative photoplethysmography, air plethysmography, and duplex quantitative valve closure time in assessing deep venous reflux. J Vasc Surg. 1992;16(6):913-9.

12. Abramowitz HB, Queral LA, Finn WR et al. The use of photoplethysmography in the assessment of venous insufficiency: a comparison to venous pressure measurements. Surgery. 1979;86(3):434-41.

13. Van Bemmelen PS, Mattos MA, Hodgson KJ et al. Does air plethysmography correlate with duplex scanning in patients with chronic venous insufficiency? J Vasc Surg. 1993;18(5):796-807.

14. Weingarten MS, Czeredarczuk M, Scovell S, Branas CC, Mignogna GM, Wolferth CC. A correlation of air plethysmography and color-flow-assisted duplex scanning in the quantification of chronic venous insufficiency. J Vasc Surg. 1996;24(5):750-4.

15. Christopoulos D, Nicolaides AN. Air plethysmography. In: Raju S, Villavicencio JL. Surgical management of venous disease. Baltimore: Williams \& Wilkins; 1997. p. 86-96.
16. Satomura S, Kaneko Z. Ultrasonic blood rheograph. Proceedings of the 3rd International Conference on Medical Electronics. 1960. p. 254-8.

17. Barnes RW, Vitti MJ. Diagnóstico não invasivo de doença venosa. In: Haimovici H, Ascer E, Hollier LH, Strandness DE Jr, Towne JB. Cirurgia vascular. $4^{\text {a }}$ ed. Rio de Janeiro: DiLivros; 1999. p.1153-70.

18. Fonseca FP. Doppler ultra-som nas doenças venosas. In Maffei FHA, Lastória S, Yoshida WB, Rollo HA, organizadores. Doenças vasculares periféricas. $3^{\text {a }}$ ed. Rio de Janeiro: Medsi; 2002. v. 1. p.471-7.

19. Menezes RE, Sales EA. Avaliação dos pacientes portadores de varizes e de insuficiência venosa crônica através do Doppler Ultra-Som. Cir Vasc Angiol. 1990;6(1):11-4.

20. Gaylis H. Some observations on peripheral venous pressure using a non-invasive technique - a preliminary report. Br J Surg. 1975; 62(4):259-63.

21. Barnes RW, Collicott PE, Mozersky DJ, Sumner DS, Strandness DE Jr. Noninvasive quantitation of venous reflux in the postphlebitic syndrome. Surg Gynecol Obstet. 1973; 136(5): 769-73

22. Barnes RW, Collicott PE, Sumner DS, Strandness DE Jr. Noninvasive quantitation of venous hemodinamics in the postphlebitic syndrome. Arch Surg. 1973; 107(5):807-14.

23. Luccas GC, Kauffman P, Lane JC. Método de avaliação do estado funcional da veia safena interna. Cir Vasc Angiol. 1985;1(3):22-8

24. Bartolo M, Antignani PL, Nicosia PM, Tidini AR. Noninvasive venous pressure measurement and its validation. Int Angiol. 1988;7(2);182-9.

25. Cheng SWK, Wong J. Chronic venous insuffiency in a chinese population: an anatomic and funcional study by continuouswave Doppler and photoplethysmography. Ann Vasc Surg. 1995;9(3):274-9.

26. Rollo HA. Avaliação do refluxo venoso profundo. In: Nectoux Filho JL, Cunha SS, Paglioli SA, Souza GG, Pereira AH. Ultra-sonografia vascular. Rio de Janeiro: Revinter; 2000. p.209-17.

27. Barnes RW, Garret WV, Hummel BA, Siamaker EE, Maixner W, Reinertson JE. Photoplethysmographic assessement of altered cutaneous circulation in the postphebitic syndrome. Proceedings of AAMI 13 th annual meeting; 197828 de março1 de abril. Washington, D.C.; 1978. p.25.

28. Evangelista SSM, Fonseca FP. O valor da fotopletismografia no pré e pós-operatório das varizes tronculares primárias dos MMII. Cir Vasc Angiol. 1996;12(Suppl. 4):59-62.

29. Gerlock AJ, Giyanani VL, Krebs C. Applications of noninvasive vascular techniques. Philadelphia: W. B. Saunders; 1988. p.181-205.

30. Sarin S, Shields DA, Farrah J, Scurr JH, Cobridge - Smith $\mathrm{PD}$. Does venous function deteriorate in patients waiting for varicose vein surgery? J R Soc Med. 1993; 86(1): 21-3.

31. Scriven JM, Bianchi V, Hartshorne T, Bell PR, Naylor AR, London NJ. A clinical and haemodynamic investigation into the role of calf perforating vein surgery in patients with venous ulceration and deep venous incompetence. Eur J Vasc Endovasc Surg. 1998; 16(2):148-52. 
32. Alvarez Sánchez JA, Vega Gómez ME. Nuevos critérios en el diagnostico de la insuficiencia venosa mediante fotopletismografia. Rev Cuba Invest Biomed. 1991;10(2):123-5.

33. Sakurai T, Matsushita M, Nishikimi N, Nimura Y. Hemodynamic assessment of femoropopliteal venous reflux in patients with primary varicose veins. J Vasc Surg. 1997;26(2):260-4.

34. Rutgers PH, Kitslaar PJ, Ermers EJ. Photoplethysmography in the diagnosis of superficial venous valvular incompetence. Br J Surg. 1993;80(3):351-3.

35. Evangelista SSM. Pletismografia no estudo das doenças venosas. In: Maffei FHA, Lastória S, Yoshida WB, Rollo HA, organizadores. Doenças vasculares periféricas. $3^{\mathrm{a}}$ ed. Rio de Janeiro: Medsi; 2002. v.1, p.479-92.

36. Guillot B. Techniques used to evaluate the cutaneous microcirculation: application of photoplethysmography to the assessment of a phlebotropic agent in the treatment of leg ulcers. Angiology. 1994;45(6):542-8.

37. Iafrati MD, Welch H, O'Donnell TF, Belkin M, Umphrey S, McLaughlin R. Correlation of venous noninvasive with the Society for Vascular Surgery/ International Society for Cardiovascular Surgery clinical classification of chronic venous insufficiency. J Vasc Surg. 1994;19(6):1001-7.

38. Tucker EA, Lamb LE, Callicott C, Cooke SD, Cooke ED. Measurements of calf muscle pump efficiency during simulated ambulation. Int Angiol. 1998;17(4):255-9.

39. Fronek A, Minn C, Kim R. Venous outflow and inflow resistance in health and venous disease. J Vasc Surg. 2000;31(3):472-6.

40. Aita DJ, Kvamme P, Rice JC, Kerstein MD. Venous insufficiency: a late sequelae of four-compartment fasciotomy in lower extremity? Am Surg. 1993;59(9):574-7.

41. Dunn RM, Fudem GM, Walton RL, Anderson FA Jr, Malhotra $\mathrm{R}$. Free flap valvular transplantation for refractory venous ulceration. J Vasc Surg. 1994;19(3):525-31.

42. Fonseca FP, Sarkis AL, Evangelista SSM. Surgery for primary troncular varicose veins without stripping the safenous vein pre and pos-operative evaluation by duplex scan and photoplethysmography. Phlebologie. 1995;(1 Suppl.):419-21.

43. Rodríguez Oliveri O, Mebold P J, Galleguillos Olmedo I. Valvuloplastia externa (banding) en pacientes con úlcera venosa de la pierna. Rev Chil Cír. 2000;52(1):73-6.

44. Lane R J, Cuzzilla ML, McMahon CG. Intermediate to long term results of repairing incompetent multiple deep venous valves using external valvular stenting. Anz J Surg. 2003; 73(5):267-74.

45. Gaitini D, Torem S, Pery M, Kaftori JK. Image directed Doppler ultrasound in the diagnosis of lower-limb venous insufficiency. J Clin Ultrasound. 1994; 22(5):291-7.

46. Sardinha WE, Miranda Jr F, Francisco Jr J, Pitta GBB, Burihan E. Avaliação da insuficiência venosa crônica pela flotopletismografia. Cir Vasc Angiol. 1988;4(3):7-11.

47. Peixoto LHWP, Braga PEG, Zorn WGW, Van Bellen B. Estase venosa e gravidez. Um estudo prospectivo fotopletismográfico. An Paul Med Cir. 1991;118(2):29-38.

48. Owens LV, Faber MA, Young ML et al. The value of air plethysmography in predicting clinical outcome after surgical treatment of chronic venous insufficiency. J Vasc Surg. 2000;32(5):961-8.
49. Comerota AJ, Harada RN, Eze AR, Katz ML. Air plethysmography: a clinical review. Int Angiol. 1995;14(1):45-52.

50. Sanches PRS. Pletismografias. Considerações básicas. In: Nectoux Filho JL, Cunha SS, Paglioli SA, Souza GG, Pereira AH. Ultra-sonografia vascular. Rio de Janeiro: Revinter; 2000. p. 24-7.

51. Kalodiki E, Nicolaides AN. Out of a recent CVI consensus some features of a basic statement. Int Angiol. 2002;21(Suppl 2):2-11.

52. Bays RA, Healy DA, Atnip RG, Neumyer M, Thiele BL. Validation of air plethysmography, photoplethysmography, and duplex ultrasonography in the evaluation of severe venous stasis. J Vasc Surg. 1994;20(5):721-7.

53. Christopoulos D, Nicolaides AN, Szendro G, Irvine AT, Bull ML, Eastcott HH. Air-plethysmography and the effect of elastic compression on venous hemodynamics of the leg. J Vasc Surg. 1987;5(1):148-59.

54. Christopoulos D, Nicolaides AN, Cook A, Irvine A, Galloway JM, Wilkinson A. Pathogenesis of venous ulceration in relation to the calf muscle pump function. Surgery. 1989;106(5):829-35.

55. Christopoulos D, Nicolaides AN, Galloway JMD, Wilkinson A. Objective noninvasive evaluation of venous surgical results. J Vasc Surg. 1988;8(6):683-7.

56. Gillespie DL, Cordts PR, Hartono C et al. The role of air plethysmography in monitoring results of venous surgery. J Vasc Surg. 1992;16(5):674-8.

57. Kalodiki E, Volteas SK, Nicolaides AN. Air plethysmography versus venography and/or duplex scanning in the diagnosis of acute deep vein thrombosis. Phlebology. 1995;(suppl 1):681-3.

58. Kalodiki E, Calahoras L, Delis KT, Zouzias CP, Nicolaides AN. Air plethysmography: the answer in detecting past deep venous thrombosis. J Vasc Surg. 2001;33(4):715-20.

59. Yang D, Vandongen YK, Stacey MC. Variability and reliability of air pletysmographic measurements for the evaluation of chronic venous disease. J Vasc Surg. 1997;26(4):638-42.

60. Engelhorn CA, Engelhorn AL, Casagrande C. Classificação da insuficiência venosa superficial baseada no Eco-Doppler colorido. In: Nectoux Filho JL, Cunha SS, Paglioli AS, Souza GG, Pereira AH. Ultra-sonografia vascular. Rio de Janeiro: Revinter; 2000. p.173-9.

61. Seidel AC. Correlação da pletismografia a ar com o quadro clínico (CEAP) e o ultra-som colorido com Doppler na insuficiência da veia safena magna [tese]. São Paulo: Universidade Federal de São Paulo, Escola Paulista de Medicina; 2003.

62. Rollo HA. Mapeamento dúplex no diagnóstico das doenças venosas. In: Maffei FHA, Lastória S, Yoshida WB, Rollo HA, organizadores. Doenças vasculares periféricas. $3^{\mathrm{a}}$ ed. Rio de Janeiro: Medsi; 2002. v. 1, p. 493-505.

63. Labropoulos N, Delis K, Nicolaides AN, Leon M, Ramaswami $\mathrm{G}$, Volteas N. The role of distribution and anatomic extent of reflux in the development of signs and symptoms in chronic venous insufficiency. J Vasc Surg. 1996;23(3):504-10.

64. Neglen P, Raju S. A comparison between descending phlebography and duplex Doppler investigation in the evaluation of reflux in chronic venous insufficiency: a challenge to phlebography as the "gold standard". J Vasc Surg. 1992;16(5):687-93. 
65. Baker SR, Burnand KG, Sommerville KM, Thomas ML, Wilson NM, Browse NL. Comparison of venous reflux assessed by duplex scanning and descending phlebography in chronic venous disease. Lancet. 1993;341(8842):400-3.

66. Delis KT, Husmann M, Kalodiki E, Wolfe JH, Nicolaides A. In situ hemodynamics of perforating veins in chronic venous insufficiency. J Vasc Surg. 2001;33(4):773-82.
Correspondência:

Orlando Adas Saliba Jr.

Departamento de Cirurgia e Ortopedia

Faculdade de Medicina de Botucatu - UNESP

CEP 18618-970 - Botucatu, SP

Tel.: (14) 3811.6269

Fax: (14) 3815.7428

E-mail: osalibajr@terra.com.br 